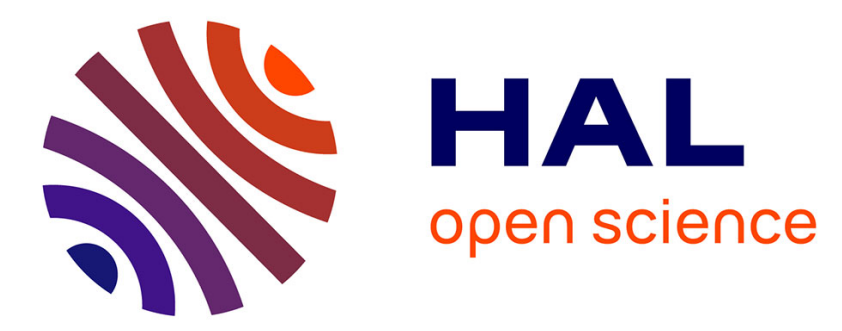

\title{
Hardness testing under a different light: combining Synchrotron X-ray Microdiffraction and Indentation techniques for polymer fibers studies
} Aurélien Gourrier, Mari-Cruz García Gutiérrez, Christian Riekel

\section{- To cite this version:}

Aurélien Gourrier, Mari-Cruz García Gutiérrez, Christian Riekel. Hardness testing under a different light: combining Synchrotron X-ray Microdiffraction and Indentation techniques for polymer fibers studies. Philosophical Magazine, 2006, 86 (33-35), pp.5753-5767. 10.1080/14786430600651988 . hal00513681

\author{
HAL Id: hal-00513681 \\ https://hal.science/hal-00513681
}

Submitted on 1 Sep 2010

HAL is a multi-disciplinary open access archive for the deposit and dissemination of scientific research documents, whether they are published or not. The documents may come from teaching and research institutions in France or abroad, or from public or private research centers.
L'archive ouverte pluridisciplinaire HAL, est destinée au dépôt et à la diffusion de documents scientifiques de niveau recherche, publiés ou non, émanant des établissements d'enseignement et de recherche français ou étrangers, des laboratoires publics ou privés. 


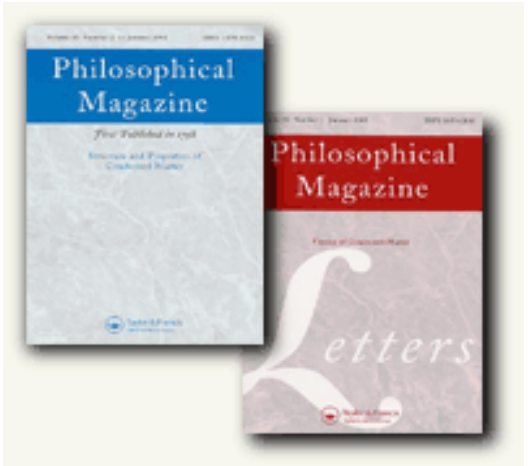

\section{Hardness testing under a different light: combining Synchrotron X-ray Microdiffraction and Indentation techniques for polymer fibers studies}

\begin{tabular}{|c|c|}
\hline Journal: & Philosophical Magazine \& Philosophical Magazine Letters \\
\hline Manuscript ID: & TPHM-05-Nov-0509.R2 \\
\hline Journal Selection: & Philosophical Magazine \\
\hline $\begin{array}{l}\text { Date Submitted by the } \\
\text { Author: }\end{array}$ & $16-F e b-2006$ \\
\hline Complete List of Authors: & $\begin{array}{l}\text { Gourrier, Aurelien; Max-Planck Institute of Colloids and Interfaces, } \\
\text { Biomaterials; ESRF, microfocus beamline ID13 } \\
\text { Garcia-Gutierrez, Mari-Cruz; CSIC, Macromecular Physics } \\
\text { Riekel, Christian; ESRF, Microfocus Beamline ID } 13\end{array}$ \\
\hline Keywords: & $\begin{array}{l}\text { texture, indentation, microstructure change, polymers, synchrotron } \\
\text { radiation, deformation mechanisms, X-ray diffraction }\end{array}$ \\
\hline Keywords (user supplied): & in-situ techniques, microbeams \\
\hline
\end{tabular}

\section{S) ScholarONE" \\ Manuscript Central}




\title{
Hardness testing under a different light: combining
}

\section{Synchrotron X-ray Microdiffraction and}

\section{Indentation techniques for polymer fibers studies}

\author{
A. GOURRIER ${ }^{\dagger}$, M.C. GARCÍA-GUTIÉRREZ ${ }^{\ddagger}$ AND C. RIEKEL ${ }^{\S}$ \\ ${ }^{\dagger}$ Max-Planck Institute of Colloids and Interfaces, Biomaterials dpt., D-14424 Potsdam, Germany \\ ${ }^{\ddagger}$ Instituto de Estructura de la Materia (CSIC), Macromolecular Physics dpt., E-28006 Madrid, Spain \\ ${ }^{\S}$ European Synchrotron Radiation Facility, B.P. 220, F-38043 Grenoble Cedex, France \\ * To whom correspondence should be addressed: email gourrier@ mpikg-golm.mpg.de; Tel +49 (0) \\ 331567 9451; Fax +49(0) 3315679402
}

\begin{abstract}
Deformation mechanisms occurring on the nanometer scale during indentation of bulk polymer fibres were investigated by means of scanning wide-angle X-ray scattering (WAXS) using beams of micrometer size at the ESRF microfocus beamline (ID13). Elastic processes could be evidenced in-situ using a dedicated microindenter device. The most important changes appear in the form of local crystalline reorientation which can be quantified by analysis of the azimuthal profiles of the WAXS reflections. The texture and phase-transitions induced by plastic deformation at higher loads were also
\end{abstract}


measured and the differences between high performance fibres and a more traditional semicrystalline polymer are emphasized.

Keywords: indentation; X-ray diffraction; deformation mechanisms; polymers

8

9

10

11

12

13

14

15

16

17

18

19

20

21

22

23

24

25

26

27

28

29

30

31

32

33

34

35

36

37

38

39

40

41

42

43

44

45

46

47

48

49

50

51

52

53

54

55

56

57

58

59

60 


\section{Introduction}

Indentation testing is a well established non-destructive technique to determine the hardness of a material by measuring the plastic deformation left by an indenter of specific shape pressed on the sample surface [1-3]. Initially used as a mean to compare different materials, it was early recognized that hardness $(H)$ is not an intrinsic property of a material but rather a complex function of other mechanical properties and experimental measuring conditions [1]. By reducing the size of the indentation this technique has become one of the most reliable methods to measure mechanical properties in a highly localized region of a sample. Under well-defined loading and unloading conditions it was recently shown that other mechanical properties such as elastic modulus (E) could be extracted on the nanoscale using specific indenter tips [4]. The results obtained e.g. from nanoindentation studies [4] have paved the way to new kinds of analysis whereby maps of the elastic modulus are obtained by indentation measurements over a region of interest of the sample [5].

However, such advances are only possible by gaining a fundamental understanding of the deformation mechanisms involved during the indentation process. In this respect, much still remains to be understood concerning the extent of the plastic flow and elastic recovery as well as the intrinsic deformation modes [6,7]. Those are, in turn, strongly dependent on the type of material and its morphology or microstructure. Several structural length scales therefore have to be considered which generally implies combining several analytical techniques. Furthermore, although precious information can be obtained by comparing the initial and deformed structures, a full understanding of the question requires real-time analysis.

Bulk in-situ methods therefore play a key role in such studies. Ideally, they should be sensitive on several length scales and have a high spatial resolution. Amongst possible techniques, Raman microspectroscopy with micron sized beams provides molecular scale information [8] but the low penetration depth of the radiation in many materials is often a strong limiting factor. Transmission electron 
microscopy (TEM) combines structural resolution on the mesoscopic scale (i.e., from about one nanometer up to a few hundred nanometers) with nanometer-sized beams. Although the combination of this technique with nanoindentation of Silicon has been demonstrated [9], very thin samples are required and organic materials generally suffer from severe radiation damage.

In polymer science, to which we restrict ourselves in the present study, the micro- and nanometer levels have been shown to play an important role in deformation processes [10]. As a consequence, microand nanoindentation have been extensively applied to polymers studies [11-13]. Due to the limitations of micro-Raman and TEM, a different route was investigated using micro X-ray scattering techniques which are bulk sensitive and require little sample preparation. Wide-angle X-ray scattering (WAXS) provides details on the atomic or molecular arrangement within crystalline regions of the specimen. Small-angle X-ray scattering (SAXS) yields complementary information on the size, shape and orientation of weakly ordered structures on the mesoscopic scale. Furthermore, high-brightness synchrotron radiation sources allow using these techniques with beam sizes in the order of 0.1-30 $\mu \mathrm{m}$. This favors position-resolved experiments and real-time studies even for poorly scattering organic samples, thus providing unique insight into the physical processes occurring in-situ [14,15].

This report shows a number of results obtained by studying microindentation on polymer fibres in-situ using synchrotron X-ray microbeams and a dedicated setup developed for this purpose at the microfocus beamline (ID13) of the European Synchrotron Radiation Facility (ESRF).

\section{Experimental section:}

\subsection{Materials}

Two high modulus (high performance) polymer fibres were investigated: ultra-high molecular weight polyethylene (UHMWPE; trade name: Dyneema ${ }^{\circledR}$ ) and a thermotropic liquid crystalline copolyester of poly-4-hydroxybenzoic acid and 2-hydroxy-6-naphtoic acid (PHBA/HNA; molar mass ration:73/27; 
trade name: Vectra A950). Those were compared with more standard semicrystalline fibres of isotactic polypropylene (iPP). Important parameters of the individual samples are given in table.1.

\subsection{Microindenter setup and sample preparation}

Single fibres were deformed using the dedicated microindenter setup specifically developed for our purposes. A detailed description has been given in a previous report [16] and is schematically shown in figure 1. The indenter tip used in this study is a square-based diamond pyramid (Vickers indenter). The samples were cut in length with micro-scissors, glued at both ends onto the diamond support and indented at room temperature $\left(25^{\circ} \mathrm{C}\right)$ under a given force $F(\mathrm{mN})$, loading rate $r\left(\mathrm{mN} . \mathrm{s}^{-1}\right)$ and holding time $t(\mathrm{~s})$. Experiments were done in either of the two following ways: (A) the samples were indented in the laboratory, removed from the diamond support, glued at the end of a glass capillary and then transfered to the beamline scanning setup for data collection or (B) were deformed in real-time, i.e. during data acquisition, using the microindenter setup directly mounted on the scanning stage. Those will respectively be referred to as static (A) and in-situ (B) experiment. The term 'indentation' refers in the following to the plastically deformed region rather than the deformation process itself and the direction of applied force will be taken as a reference $\left(0^{\circ}\right.$ or parallel direction). While in-situ experiments currently only allow viewing the sample normal to the indentation (dotted arrow in figure

1 (a), virtually any direction can be used in case of static analysis. Most frequently, however, scans were performed either normal to the indentation (as in in-situ studies) or parallel (in the direction of applied load).

\subsection{Synchrotron Radiation Experiments}

The experiments were carried out at the microfocus beamline (ID13) of the European Synchrotron Radiation Facility (ESRF) [14]. A monochromatic beam of wavelength $\lambda=0.097_{6} \mathrm{~nm}(E=12.78 \mathrm{KeV})$ was first focused with an ellipsoid mirror and further reduced in size with either a tapered glass 
capillary $(3 \mu \mathrm{m})$ or collimators $(5 \mu \mathrm{m})$ as shown schematically in figure 2 . By mounting the samples on a motorized XYZ translation stage, position resolved measurements could be achieved by displacing the sample with respect to the beam. This experimental technique allows combining the local scan resolution defined by the size of the beam with the reciprocal space resolution of SAXS/WAXS techniques. Two different methods were used for the experiments described in this report, depending on whether static or in-situ experiments were considered. In the first case, the deformed sample was scanned in the beam and SAXS/WAXS patterns were collected at each point. In the latter case, the sample was placed at a fixed position with respect to the beam, and data were collected as a function of time during the indentation cycle. A slow-scan 16 bit-readout CCD detector (X-ray Associates; XRA) with an X-ray converter screen of $130 \mathrm{~mm}$ diameter and $2048 \times 2048$ pixels of $64.45 \times 64.45 \mu \mathrm{m}^{2}$ was used for static experiments data collection with a readout time of $12 \mathrm{~s} /$ frame. For in situ studies a faster readout (about $1.5 \mathrm{~s} /$ frame) 12 bit-readout Gemstar detector (PHOTONIC) was also used. The total exposure time was optimized for each sample and will be mentioned in the text when appropriate. The sample to detector distance and the center of the detector were calibrated using an Ag-behenate standard [17] and the data were analyzed using the FIT2D software package [18].

\subsection{Calibration}

The microindenter setup was calibrated in order to correlate the positions of the X-ray micro-beam and of the diamond tip. A GaN microcrystal showing luminescence in the visible range when exposed to Xrays was glued at the end of a sharp glass tip and positioned at the focal spot of the objectives of the setup. The device was then translated at exact beam position with micrometer precision by monitoring the light intensity emitted by the GaN. The microcrystal was subsequently replaced by a Kapton® foil glued on the surface of the diamond sample-holder. The position of an indentation of $350 \mathrm{mN}$ at 35

$\mathrm{mN} . \mathrm{s}^{-1}$ with $10 \mathrm{~s}$ hold time was then matched with the position of the beam. Samples could then 
reproducibly be indented and exposed to X-rays with a precision of $\pm 1 \mu \mathrm{m}$ laterally and vertically using fibres with diameters down to $10 \mu \mathrm{m}$.

\section{3 - Results and Discussion}

Selected WAXS patterns of native UHMWPE, iPP and PHBA/HNA fibres are shown in figure 3 (a), (b), (c). The crystalline fractions are reflected by Bragg peaks, while the diffuse halo observed for PHBA/HNA results from disorder due to its liquid-crystalline nature. All patterns show fibre texture, which facilitates data analysis and has led to the choice of these samples as model systems for the insitu microindenter setup. Only the equatorial reflections shown in figure 3 (d), (e), (f) will be considered for further analysis with particular focus on the $110_{\mathrm{O}}, 110_{\mathrm{M}}$ and $110 / 200_{\mathrm{PH}}$ peaks of respectively UHMWPE, iPP and PHBA/HNA. The subscripts $\mathrm{O}$ stands for orthorhombic, $\mathrm{M}$ for monoclinic and PH for pseudo-hexagonal crystalline systems used to index the reflections as described in detail in previous reports $[16,19,20]$. In addition, a full analysis of all parameters of the scattering pattern being beyond the scope of this report, the results presented in the following will mostly detail the evolution of the peaks along the azimuth (shown in figure 3 (a)). For this purpose, the azimuthal profile of a peak is calculated by projecting the peak intensity along the radial direction onto the azimuth. The center of this $1 \mathrm{D}$ profile is generally determined by peak fitting and gives a measure of the mean crystalline orientation within the volume probed by the beam while the full width at half maximum (FWHM) gives an indication of the orientation distribution about this average value.

\subsection{In-situ experiments}

UWMW-PE and iPP were indented following the schemes of figure 4 (a), (b). The fibres were respectively indented up to $5 \mathrm{mN}$ and $30 \mathrm{mN}$ at $1 \mathrm{mN} \cdot \mathrm{s}^{-1}$ with a holding time of $10 \mathrm{~s}$ and exposed for $0.5 \mathrm{~s}$. The $5 \mu \mathrm{m}$ diameter X-ray beam was centered in each case below the indenter tip with an 
accuracy of $\pm 0.5 \mu \mathrm{m}$ and positioned at $8 \mu \mathrm{m}$ below the top of the fibres. Additional time-scans were collected for iPP with a beam laterally displaced in steps of $7 \mu \mathrm{m}$ (figure 4 (b)).

The evolution of the $110_{\mathrm{O}}$ UHMWPE reflection is shown as a function of time in figure 5 (b) at different positions of the loading cycle (figure 5 (a)). The reflection is observed to broaden along the azimuth and split at maximum load before nearly recovering its original shape upon retraction of the indenter tip. This is further emphasized in the azimuthal profiles shown in figure 5 (c) at selected positions of the indentation cycle, i.e., before deformation, at maximum load and at the end of the indentation cycle in the relaxed state. At the onset of deformation and after relaxation, the profile can be fitted using a single Gaussian function whereas two are necessary during loading. The increase of the reflection width (full-width-at-half-maximum; FWHM) is determined to be $1.5^{\circ}$ in the relaxed state and to $\sim 9^{\circ}$ at maximum load (sum of the FWHM of the two peaks). Since this phenomenon is reversible, it clearly suggests an elastic deformation process where the local crystalline orientation is temporarily perturbed [16].

Similar observations can be made concerning the evolution of the $110_{\mathrm{M}}$ reflection of the indented iPP fibre immediately below the indenter tip [19] (within dashed rectangle in figure 6 (b)). The peak also broadens along the azimuthal direction before recovering its original position, thus suggesting the presence of an elastic process. This is better seen in the corresponding profiles (figure 6 (c)) at selected positions of the indentation cycle (figure 6 (a)), namely at the initial stage of deformation, at maximum load and after completion of the indentation cycle. Unlike for the highly crystalline UHMWPE where the molecules are close to fully extended, two Gaussian functions are necessary to fit the azimuthal profiles of the initial and final states. Those two functions account for both the Bragg reflection and the diffuse background scattering produced by the amorphous phase. At maximum load, a clear symmetrical splitting of the peak is not observed as for UHMWPE. An additional Gaussian is needed to obtain a correct fit (central profile in figure $6(\mathrm{c})$ ) although its extent $\left(\mathrm{FWHM} \sim 4^{\mathrm{o}}\right.$ ) is considerably less than in UHMWPE. In addition, it is noteworthy that the shift along the azimuthal direction of the 
most intense peak corresponding to the Bragg reflection is insignificant with respect of the broadening effect. This suggests that only a fraction of the crystalline domains probed in the volume of the beam actually deforms elastically while a fraction of the crystalline domains retains its initial orientation. The latter corresponds to the main peak in the azimuthal profile while the former has to be accounted for by the additional Gaussian function. The asymmetry observed in the profile is due to a slight offset of the beam position with respect to the exact position of the indenter tip as will be discussed below.

The temporal variation of the $110_{\mathrm{O}}$ reflection measured with the beam displaced in $7 \mu \mathrm{m}$ steps along the fibre axis (figure 6 (b)) reveals additional information concerning the extent of the elastic deformation. The reference position of the indenter tip is indicated by $0 \mu \mathrm{m}$. The reflection is also seen to broaden at -7 and $7 \mu \mathrm{m}$, on both sides of the tip. However, the effect gradually fades away from 0 to $14 \mu \mathrm{m}$, where little changes are observed in the peak profiles. In reality, the extent of the deformation can even be reduced to $11.5 \mu \mathrm{m}$ taking into account the extent of the beam radius $(2.5 \mu \mathrm{m})$. These experiments show that both the intensity and the extent of the plastic deformation can be measured insitu in a qualitative and quantitative way using this technique..

\subsection{Static experiments}

In order to better understand the observations made above, static experiments were performed whereby the same fibres were indented up to $10 \mathrm{mN}$ for UHMWPE and $40 \mathrm{mN}$ for iPP at $1 \mathrm{mN} \cdot \mathrm{s}^{-1}$ with a holding time of $10 \mathrm{~s}$ at room temperature $\left(25^{\circ} \mathrm{C}\right)$. Once indented, those were scanned in a $5 \mu \mathrm{m}$ beam following the scheme of figure 7 (a), (b) with $5 \mu \mathrm{m}$ steps along the indentation axis and $10 \mu \mathrm{m}$ along the fibre axis and exposed for $5 \mathrm{~s}$. Since the whole region was not mapped, care was taken that the central vertical scan was taken in the middle of the indentation. Figure 8 (a) shows a composite image of the three equatorial peaks described in figure 3 (a), (d) at each scan point within the vicinity of the indentation. Due to convolution of the beam and fibre diameter (see figure 7 (a), the sample appears larger and is thus represented by dashed line on the figure. The approximate position of the center of 
the indentation is also shown to facilitate interpretation. The first observation is a clear broadening of the $110_{\mathrm{O}}$ reflection, particularly in the lower two lines. The azimuthal profile of the centermost frame shown in figure 8 (b) reveals two satellite peaks about the central most intense one separated by $\sim 19^{\circ}$ at maximum. From the previous observations of the in-situ experiments, it can be thought that the central and most intense peak corresponds to material that has either been deformed elastically and recovered its original orientation or has not been deformed at all. The two peaks on either side, however, clearly indicate a permanent deformation of at least a fraction of the crystallites in two domains of preferred orientation at $\sim 9.5^{\circ}$ from the fibre axis. In other words, the plastic deformation induces some degree of texture in terms of crystalline orientation within the material. A second important observation is the presence of a more intense $001_{\mathrm{M}}$ peak particularly visible in the second line from the top of the scan. This metastable monoclinic phase is a clear signature of a partial phase transition occurring in the vicinity of the indenter tip. Detailed analysis of the distribution of this phase is beyond the scope of this report, but it was shown that the texture induced by the indentation process can be quantitatively described considering the broadening of this particular peak [21]. This follows the observation that this phase essentially forms during the indentation process as opposed to the orthorhombic form. In the latter case, care must be taken to separate the peak corresponding to the undeformed fraction of the material and those originating from the permanent reorientation of a part of the crystalline domains. A similar composite image of the first three most intense equatorial reflections of iPP in the vicinity of the indentation is shown in figure 9 (a). Since the fibre is much larger in diameter, only the upper part is shown schematically by the dashed line indicating $\mathrm{z}=0 \mu \mathrm{m}$. The azimuthal profiles of the $110_{\mathrm{M}}$ reflection obtained in the center of the indentation and well away (at 15 $\mu \mathrm{m}$ across and $30 \mu \mathrm{m}$ along the fibre axis) are shown respectively in the upper and lower part of figure 9 (b). As previously, the two Gaussian functions necessary to fit the latter to account for the crystalline and amorphous fraction of the material are no longer sufficient in the deformed area. Two extra functions separated by $\sim 12^{\circ}$ at maximum are necessary in addition to the main crystalline peak, 
although the separation is less obvious in this case than in UHMWPE. Nevertheless, this also indicates a permanent deformation in the form of a partial reorientation of the crystallites in two preferred orientations at $\sim 6^{\circ}$ from the average orientation of the undeformed fibre.

The last scan presented in figure 10 adds important information to this discussion. The scan was performed with a PHBA/HNA fibre using a $2 \mu \mathrm{m}$ beam which significantly increases the resolution in direct space (by a factor of 2.5 with respect to the previous experiments). This allows a much finer mapping of the fibre as shown schematically in figure 11 (a). In particular, an optimum resolution was chosen in vertical direction, i.e. across the fibre, using scan steps of $2 \mu \mathrm{m}$. The composite image shown in figure 10 (a) of the equatorial reflections detailed in figure 3 (f) reveals that the peaks tend to tilt in the vicinity of the indentation. The broadening effect along the azimuth direction observed for UHMWPE and iPP, although also observed in this case (1-2 ${ }^{\circ}$ at maximum), is however very weak as compared to the shift of the peaks along the azimuth $\left(\sim 5^{\circ}\right.$ at maximum). Detailed analysis reveals that the tilt is observed for the whole pattern in a correlated way, which implies that the whole of the crystalline domains probed within the beam tend to re-orient under the stress field of the indenter. This is further emphasized in figure 10 (b) showing a detailed area of the scan immediately under the tip where the vectors indicate in a qualitative way the tilt of the diffraction pattern. A scan with the same parameters was performed along the direction of the indentation (figure 11 (b)) and detailed analysis was performed to measure the shift and broadening of the $110_{\mathrm{PH}}$ reflection along the azimuth. The result for both orientations is represented in the form of a vector plot in figure 11 (c), (d). The length and orientation of the vectors at each scan point are respectively proportional to the full-width at halfmaximum (FWHM) of the azimuthal peak profile and to the tilt along the azimuth. This allows experimental reconstruction of a $2 \mathrm{D}$ projection map of the crystalline orientation within the fibre which is qualitatively indicated by the dotted lines, and shows that a 3D reconstruction should, in principle, be feasible. Equally important for this sample is the observation of a complex crystalline phase transition from a pseudo-hexagonal to a mixture of two orthorhombic phases which was described in detail in a 
previous report [20]. The corresponding maps of the integrated intensity of the most intense peak of the new phase for each orientation are therefore displayed in figure 11 (e), (f). Although in our case not yet feasible, if the precise nature and value of the stress needed to induce such phase transition was known, e.g. by performing compressive or shear experiments, a $2 \mathrm{D}$ and possibly 3D map of the stress field could therefore experimentally be reconstructed.

From the above results obtained in-situ and during static experiments, a general description can now be derived concerning the deformation mechanism involved in the indentation process based on microstructural considerations. First, it is important to recall that in all cases, at least a part of the crystalline domains tend to reorient in the stress field of the indenter giving rise to some degree of texture. The essential differences observed in the three samples lie in the extent of the azimuthal broadening or splitting of the peaks and their degree of tilting with respect to the average direction. In order to better understand the origin of those changes, several cases must be considered for which a simplified 2D model will be used. It is assumed, in first approximation that the crystalline domains would reorient parallel to the faces of the diamond tip during the deformation process as schematically shown in figure 12 (a). If the beam is sufficiently small to probe a single orientation (case 1 or 2 in figure 12 (a)), the corresponding pattern will simply appear to have tilted with respect to the initial undeformed fibre axis (figure 12 (b), (c)) as observed for the case of PHBA/HNA. If, on the other hand, the beam is large enough to probe several orientations at the same time (case 3 in figure 12 (a)), the pattern will result in a superposition of the scattering from all crystalline directions. Hence, the peaks will be observed to broaden along the azimuthal direction and ultimately split above a critical tilt (figure 12 (d), (e)) as for iPP and UHMWPE. From this, it can therefore be inferred that all the changes observed due to the indentation process seem to originate from a local rearrangement of the crystalline domains along the faces of the indenter tip. In addition, quantitative analysis allowing to deconvolute the signal of the undeformed and deformed fraction of the material is only possible providing the beam 
size is below a critical length scale which could be dependent on either or both of the following factors : the experimental geometry and the nature of the material. The iPP fibres are $28 \mu \mathrm{m}$ in diameter as opposed to $12 \mu \mathrm{m}$ for UHMWPE and the beam is at $8 \mu \mathrm{m}$ from the top of the fibre in both cases which gives a relative position of respectively $\sim 0.29$ and $0.67(8 / 28$ and $8 / 12)$.This should result (for a given indentation load) in a broader extent in iPP for which the beam is closer to the deformation zone than in UHMWPE which is the opposite of what is found. Moreover, the indentation force to fibre diameter ratio $\left(\mathrm{F}_{\mathrm{i}} / \mathrm{D} \mathrm{f}\right)$ is higher in iPP than UHMWPE (respectively $~ 1.07$ and $0.42 \mathrm{mN} . \mu \mathrm{m}-1$ ), which again is in contradiction with the observations. This tends to suggest that the most important parameter to consider lies in the nature of the polymers, i.e. in their microstructure. Further investigations should therefore be conducted to assess the length-scale parameters for the different samples, bearing in mind that some authors suggest that the nanofibril level should be given particular attention in deformation studies of polymers [10]. Those results should, in turn, be compared to complementary fundamental structural investigations of other materials [22].

\section{Conclusion}

Using scanning synchrotron radiation X-ray scattering, it was possible to study the deformation processes occurring on a nanoscopic scale during indentation in polymer fibres. For this purpose, a microindentation setup was developed to allow simultaneous microdiffraction acquisition while deforming the samples. The results presented in this report demonstrate how the analysis of the azimuthal profiles of the diffraction peaks yield information concerning reorientation mechanisms of crystalline domains within the fibres. A strong texture is induced by the deformation process, which can be evidenced by scanning indented fibres (plastically deformed) in different orientations with respect to the beam. This texture can be described quantitatively by measuring the tilt of the diffraction pattern or the peak broadening and splitting along the azimuth. In this way, it was shown that $2 \mathrm{D}$ 
projection maps of the strain field can be reconstructed and compared in some cases with images of phase transformation induced by the indentation process. In-situ experiments performed on several fibres show that it is also possible to observe elastic deformation mechanisms and quantify its extent. The most critical parameter for such experiments is clearly the size of the X-ray beam, which has to be sufficiently small to allow deconvolution of the peaks originating from the deformed and undeformed fraction of the material. Furthermore, it seems that this is in turn dependent on the intrinsic nature of the material and its microstructure since the extent of the changes varies with the different samples. In other words, the critical parameter is the relative size of the beam with respect to a given microstructural parameter, which yet has to be determined. 


\section{References}

[1] D. Tabor, The hardness of metals (Clarendon Press, Oxford, 1951)

[2] B.W. Mott, Microindentation hardness testing (Butterworths Scientific Pub., London, 1956)

[3] H. O'Neill, Hardness measurements of metals and alloys (Chapman and Hall, London, 1967)

[4] W.C. Oliver, G.M. Pharr, J. Mater. Res. 71564 (1992)

[5] J.F. Graham, V. McCague, O.L. Warren, P.R. Norton, Polymer Communications 414761 (2000)

[6] D. Tabor, Phil. Mag. A74 1207 (1996)

[7] L. Lin, A.S. Argon, J. of Mater. Sci. 29294 (1994)

[8] Y. Gogotsi, T. Miletich, M. Gardner, M. Rosenberg, Rev. Sci. Instr. 704612 (1999)

[9] M.A. Wall, U. Dahmen, Micro. Res. Tech. 42248 (1998)

[10] L.C. Sawyer, and D.T. Grubb, Polymer microscopy (Chapman \& Hall., London, 1996)

[11] F.J. Baltà-Calleja, S. Fakirov, Microhardness of polymers (Cambridge University Press, 2000)

[12] J.L. Loubet, J.M. Georges, O. Marchesini, G. Meille, trans. ASME J. tribol. 10643 (1984)

[13] M.R. VanLandingham, J.S. Villarrubia, W.F. Guthrie, G.F. Meyers, Macromol. Symp. 16715 (2001)

[14] C. Riekel, Rep. Prog. Phys. 63233 (2000)

[15] C. Riekel, R. Davies, Cur. Op. Coll. Int. Sci. 9396 (2005) 
Philosophical Magazine \& Philosophical Magazine Letters

Page 16 of 31

1
2
3
4
5
6
7
8
9
10
11
12
13
14
15
16
17
18
19
20
21
22
23
24
25
26
27
28
29
30
31
32
33
34
35
36
37
38
39
40
41
42
43
44
45
46
47
48
49
50
51
52
53
54
55
56
57
58
59
60

[16] A. Gourrier, M.C. Garcia-Gutierrez, C. Riekel, Macromolecules 358072 (2002)

[17] T.N. Blanton, et al., Powder Diffr. 1091 (1995)

[18] A.P. Hammersley, ESRF Internal Report; Report No. ESRF97 HA02T; European Synchrotron Radiation Facility: Grenoble Cedex, France (1997)

[19] M.C. Garcia-Gutierrez, A. Gourrier, C. Riekel, J. Macro. Sci. B43 267 (2004)

[20] A. Gourrier, M.C. Garcia-Gutierrez, C. Riekel, Macromolecules 383838 (2005)

[21] C. Riekel, M.C. Garcia-Gutierrez, A. Gourrier, S. Roth, Anal. Bioanal. Chem. 376594 (2003)

[22] W. Yang, B.C. Larson, G.M. Pharr, G.E. Ice, J.D. Budai, J.Z. Tischler, W. Liu, J. Mater. Sci. 19 66 (2004)

http://mc.manuscriptcentral.com/pm-pml 


\section{Tables}

1. Materials properties of polymer fibres used in the present study. (source : Goodfellow Ltd)

\begin{tabular}{|c|c|c|c|c|c|c|}
\hline & $\begin{array}{c}\text { Fibre } \\
\text { diameter } \\
(\mu \mathrm{m})\end{array}$ & $\begin{array}{c}\text { Molecular } \\
\text { weight } \\
\text { Mw x 10 } \\
\left(\text { g.mol }^{-1}\right)\end{array}$ & $\begin{array}{c}\text { Density } \\
\qquad\end{array}$ & $\begin{array}{c}\text { Crystallinity } \\
\qquad \chi_{c}\end{array}$ & $\begin{array}{c}\text { Glass } \\
\text { transition } \\
\text { temperature } \\
\mathbf{T}_{\mathrm{g}}\left({ }^{\circ} \mathrm{C}\right)\end{array}$ & $\begin{array}{c}\text { Hardness } \\
\text { (MPa) }\end{array}$ \\
\hline UHMWPE & 12 & $3000-6000$ & 0.94 & $0.85-0.95$ & & 153 \\
\hline HBA/HNA & 23 & unknown & 1.4 & $0.17-0.26$ & $50-150$ & 216 \\
\hline PP & 28 & 280 & 0.9 & $0.45-0.6$ & -10 & 76 \\
\hline
\end{tabular}




\section{Figure captions}

Figure 1 Microindenter setup design (a) : the sample is glued on a diamond support (c) mounted on an XYZ translation stage which allows positioning with respect to the Vickers diamond tip (red arrow in (c)). A region of interest can be selected online using a set of microscope objectives fixed on the rotating turret (b) and a CCD camera. The device can be mounted on the beamline translation stage for micro X-ray scattering experiments (the beam direction is indicated in (a) by a dotted arrow). Calibration of the X-ray beam position is obtained by replacing the sample-holder by a GaN crystal.

Figure 2 Scheme of a scanning X-ray scattering experiment: a sample mounted on a translation stage is scanned in a beam of micrometer size obtained by focussing and/or collimating optics. The scattering pattern is recorded on a CCD camera. A region of interest can be selected by translating the sample in the axis of a video microscope. The microindenter setup shown in fig.1 can be mounted directly on the translation stage.

Figure 3 Selected WAXS patterns of undeformed (a) UHMWPE, (b) iPP and (c) PHBA/HNA fibres. The meridional direction corresponds in this case to the fibre axis. Details of the equatorial reflections are shown respectively in (d), (e), (f) along with the corresponding crystalline indexes; O, M and PH: orthorhombic, monoclinic and pseudo-hexagonal.

Figure 4 Scaled schematic graphic of the in-situ experiments: the UHMWPE (a) and iPP (b) fibres were indented up to $5 \mathrm{mN}$ and $30 \mathrm{mN}$ (at $1 \mathrm{mN} . \mathrm{s}^{-1}$ for $10 \mathrm{~s}$ ) during data collection $(0.5 \mathrm{~s}$ exposure time) with a beam of $5 \mu \mathrm{m}$. For iPP, the experiment was repeated with a beam displaced by $7 \mu \mathrm{m}$ along the fibre axis with respect to the indenter tip position.

Figure 5 Evolution of the UHMWPE $110_{\mathrm{O}}$ reflection (b) as a function of the loading cycle (a). The azimuthal profiles (c) are calculated, from top to bottom, before deformation, at maximum 
pressure and upon removal of the load.

Figure 6 Evolution of the iPP $110_{\mathrm{M}}$ reflection (b) as a function of the loading cycle (a) and relative beam position with respect to the indenter tip and corresponding azimuthal profiles (c).

Figure 7 Scaled schemes of the static experiments on UHMWPE (a) and iPP (b). The fibres were indented up to respectively $10 \mathrm{mN}$ and $40 \mathrm{mN}$ (at $1 \mathrm{mN} \cdot \mathrm{s}^{-1}$ for $10 \mathrm{~s}$ ) and subsequently scanned online with a $5 \mu \mathrm{m}$ beam ( $5 \mathrm{~s}$ exposure time) in steps of $5 \mu \mathrm{m}$ across and $10 \mu \mathrm{m}$ along the fibre.

Figure 8 Composite image of the main equatorial reflections of UHMWPE at each scan position (a) and azimuthal profile of the $110_{\mathrm{O}}$ reflection in the center of the indentation (b).

Figure 9 Composite image of the most intense equatorial peaks of iPP as a function of scan position (a) and azimuthal profiles (b) taken in the center of the indentation (top) and in an undeformed region (bottom).

Figure 10 Composite image of the equatorial reflections of pHBA/HNA at scanned positions (a). A vector is used to represent the tilt of the scattering pattern (b).

Figure 11 Schemes of the scanning experiment on pHBA/HNA normal (a) and parallel (b) to the direction of indentation. A representation of the vector described in figure 10(b) is used to emphasize the tilt of the scattering pattern at each scanned position (c),(d). A phase transformation is observed and its extent is shown in (e),(f).

Figure 12 Schematic diagram of the possible results of orientation changes on the diffraction pattern. (b), (d) correspond to case 2 (single crystalline orientation) in the simplified 2D model shown in (a) and (c), (e) result from the superposition of several crystalline directions (case 3). 


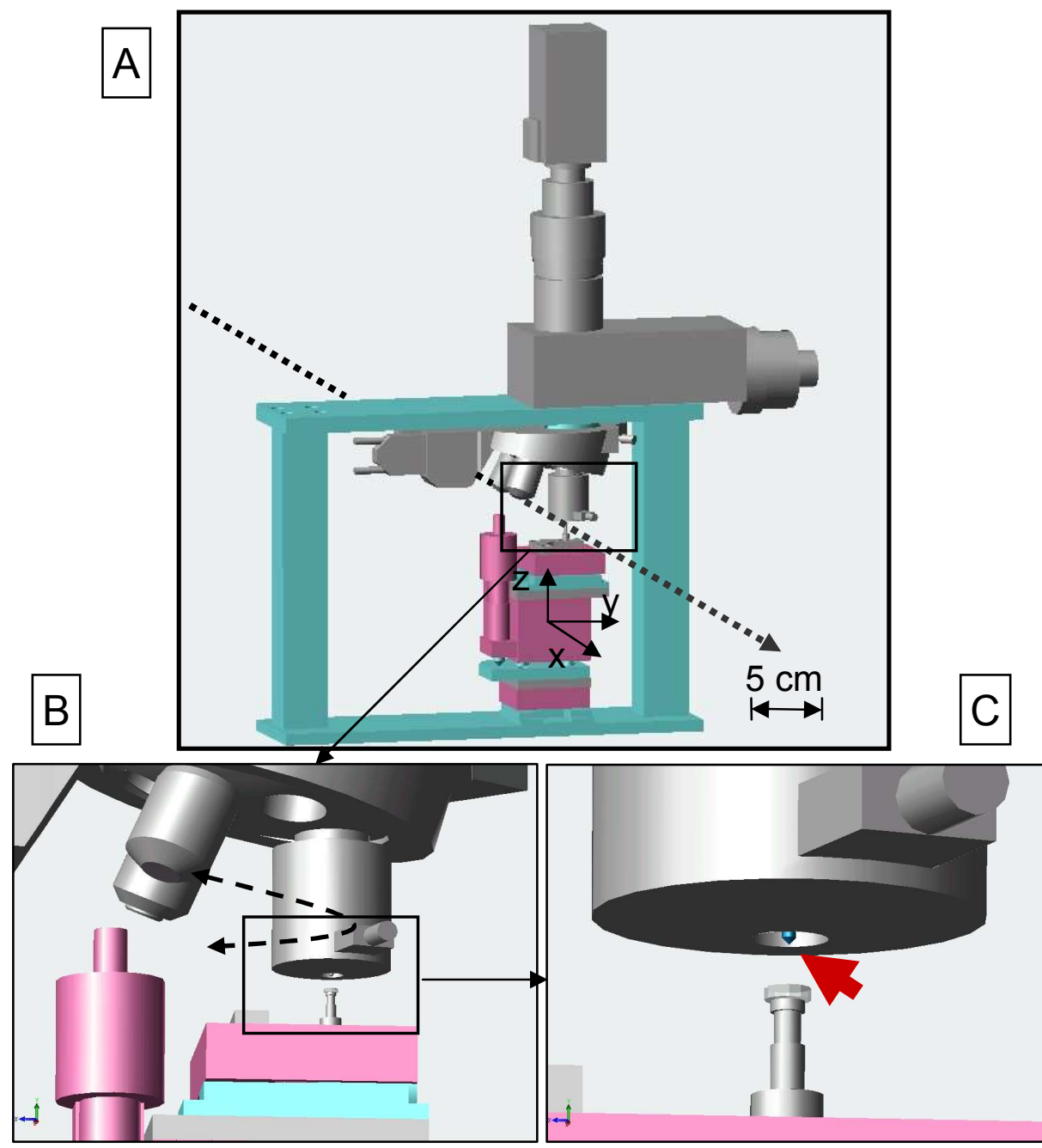

http://mc.manuscriptcentral.com/pm-pml 


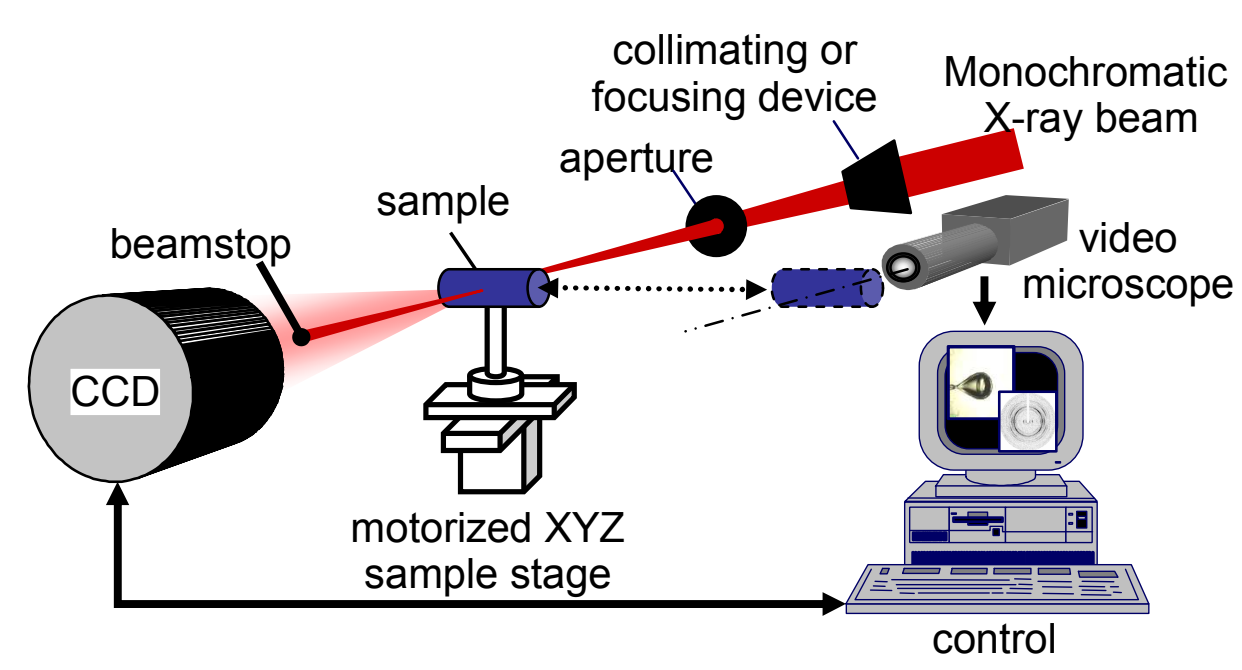




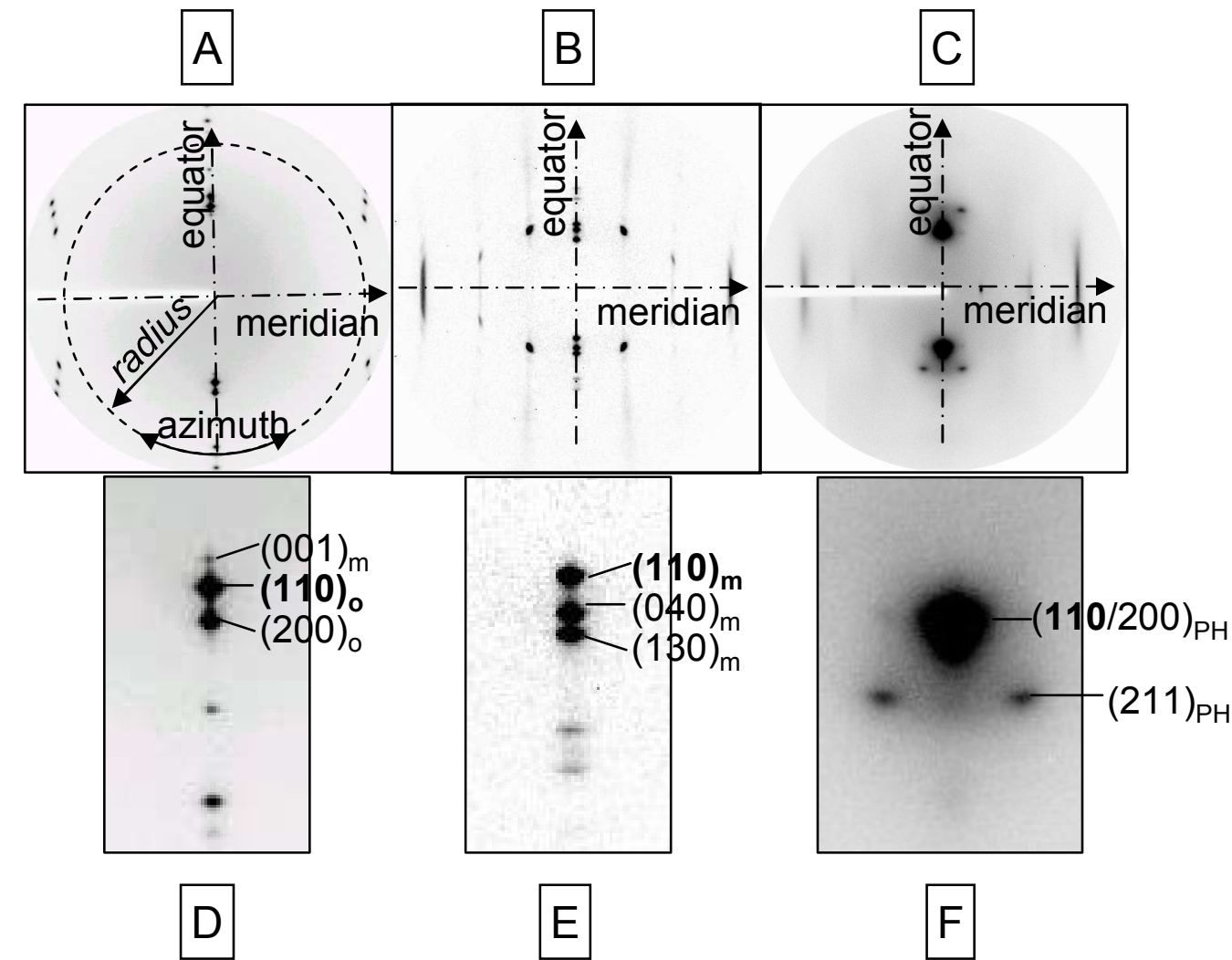



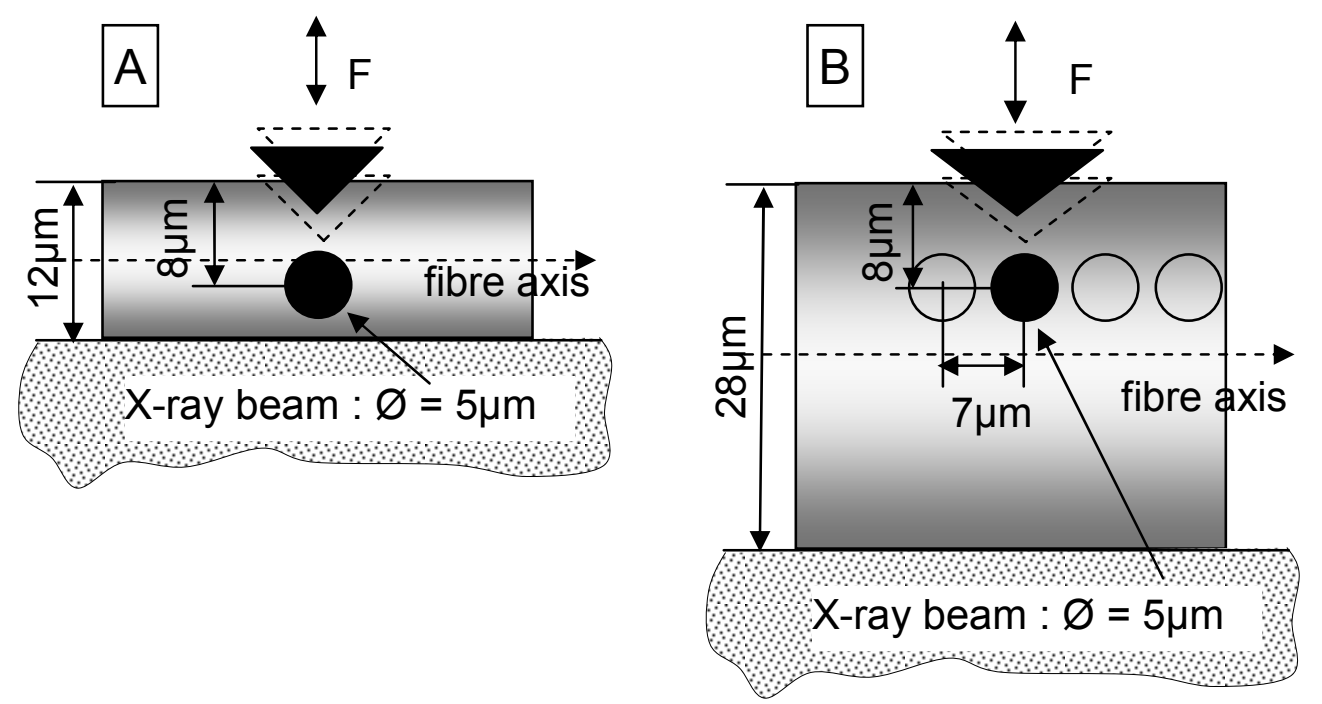


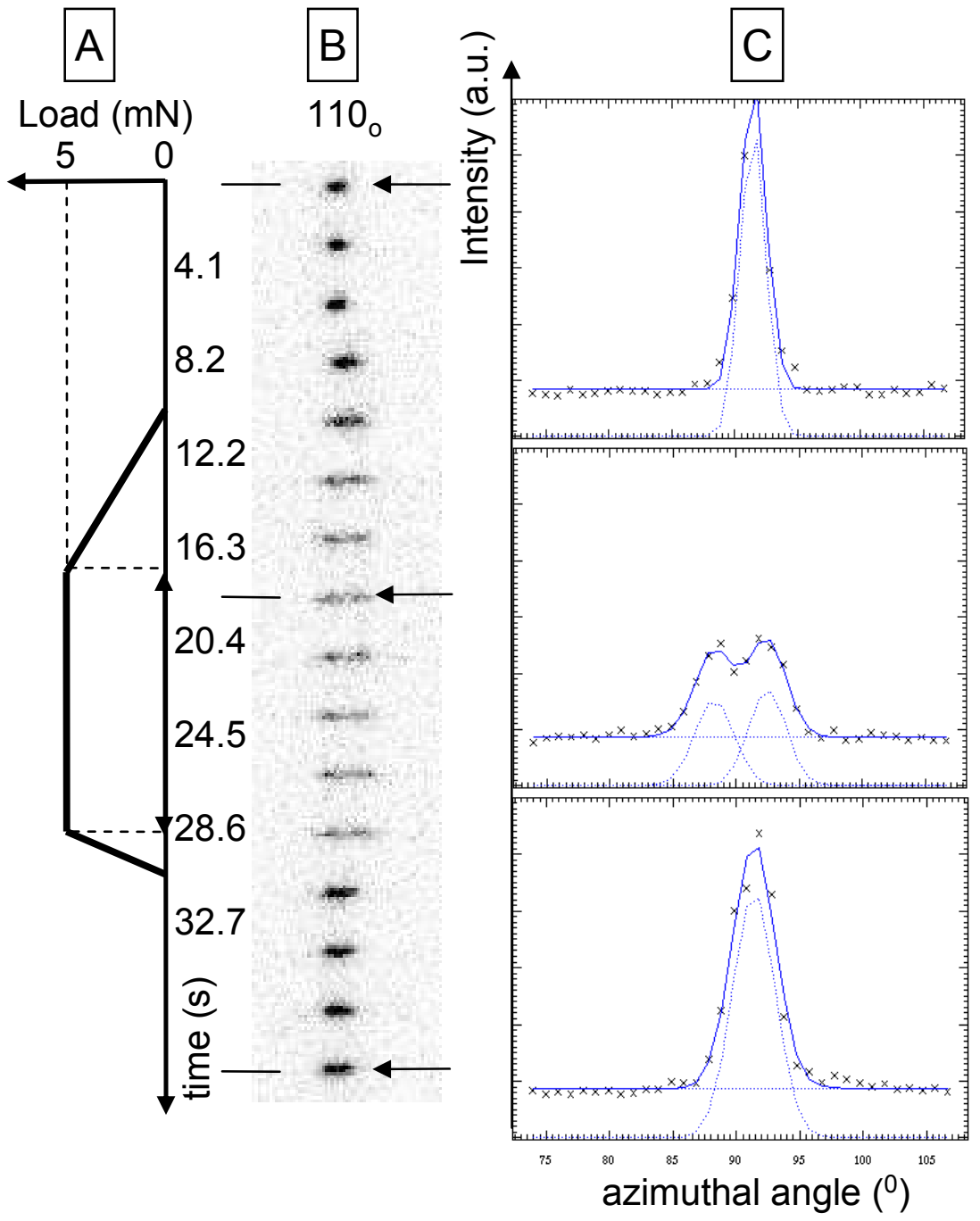

http://mc.manuscriptcentral.com/pm-pml 


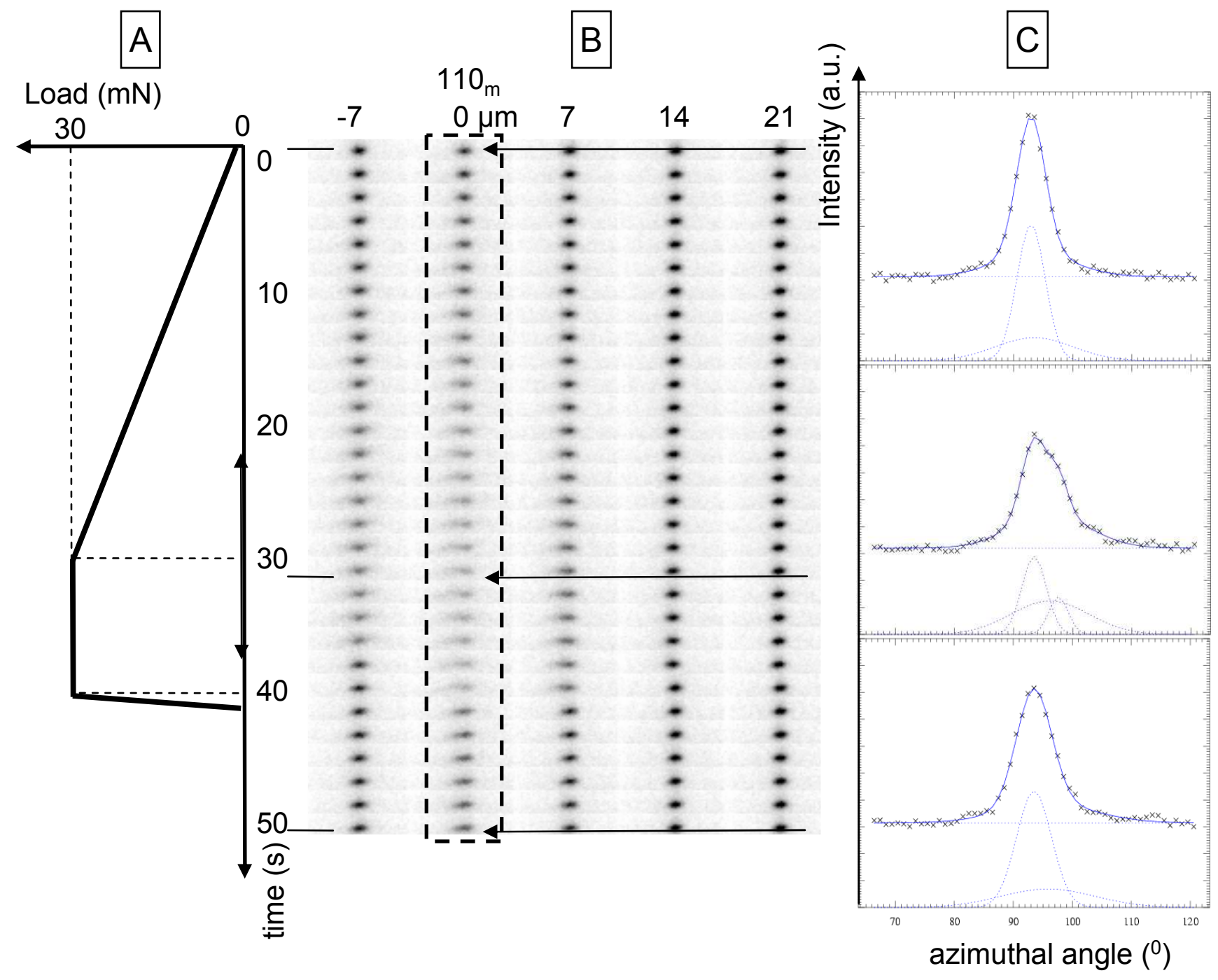




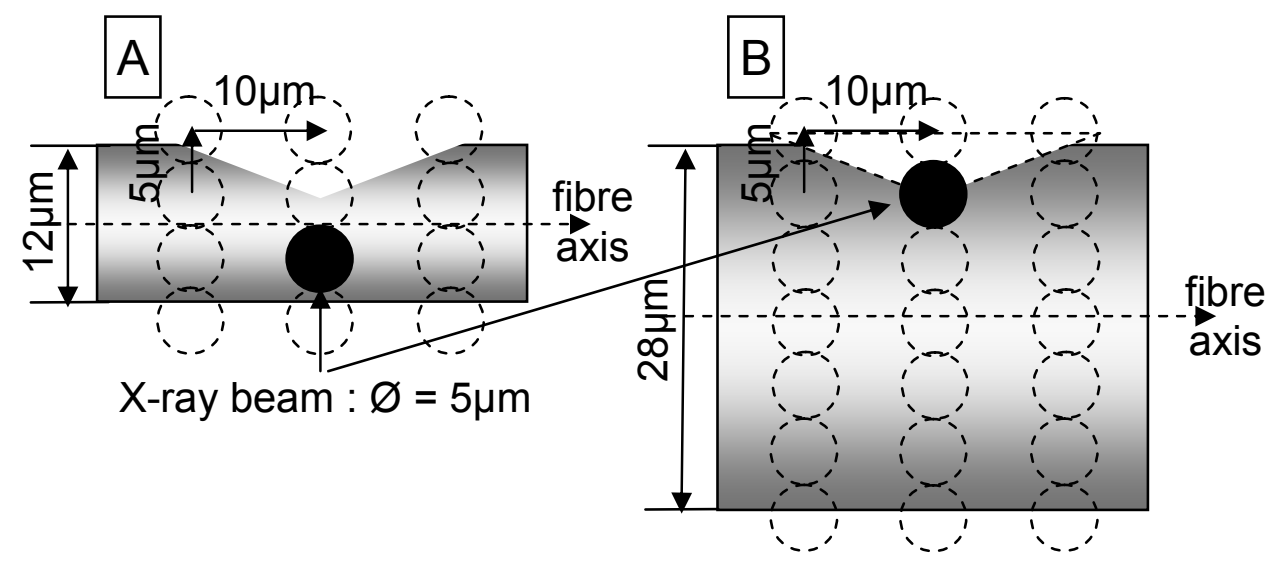




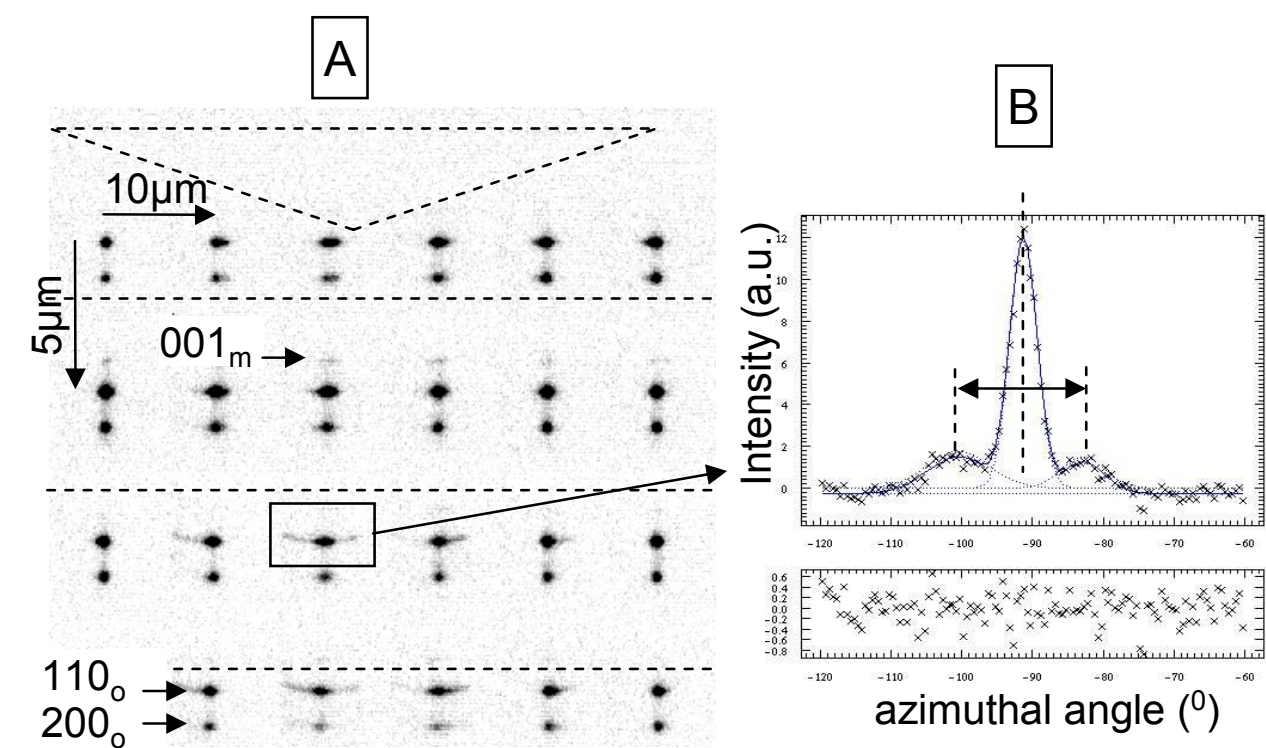




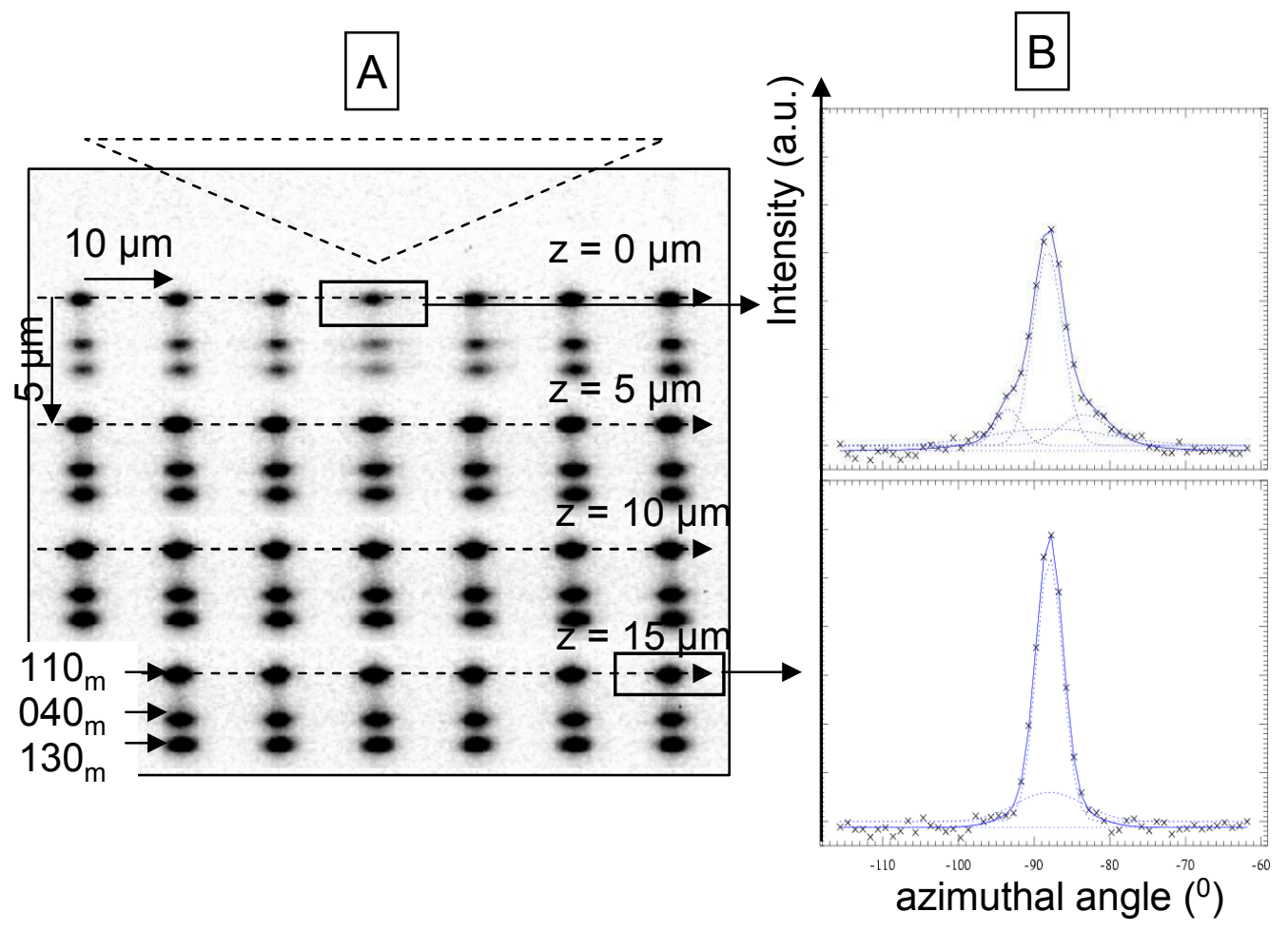

http://mc.manuscriptcentral.com/pm-pml 


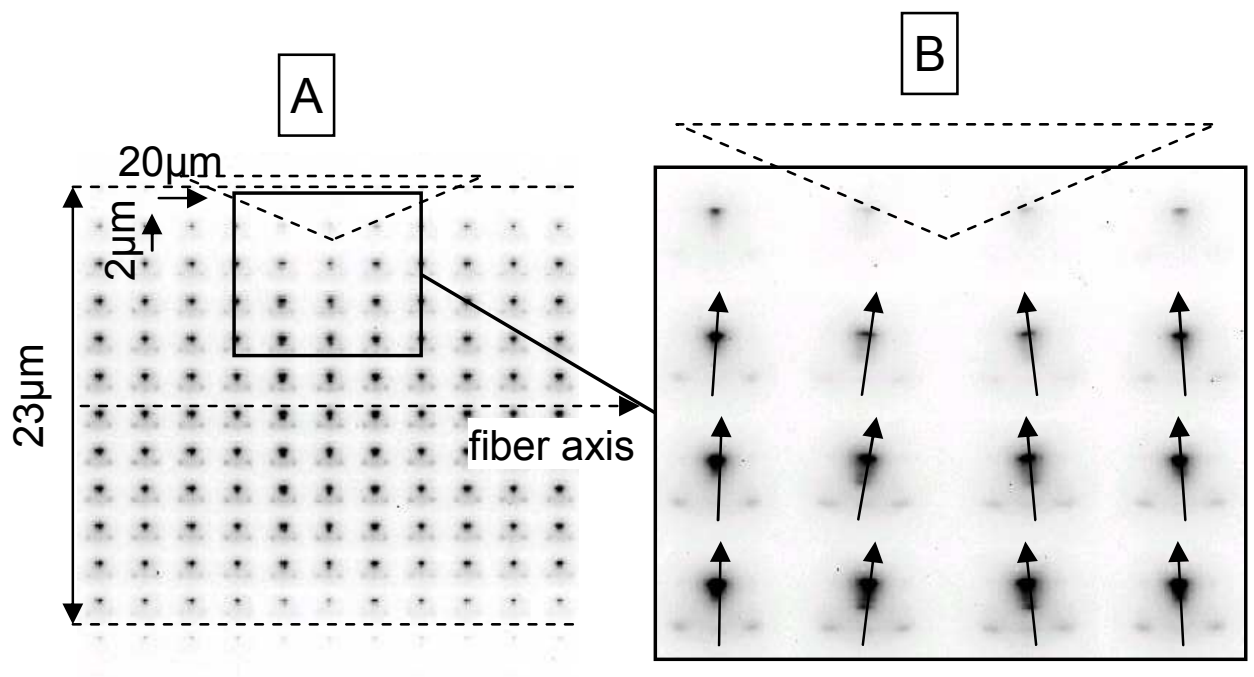



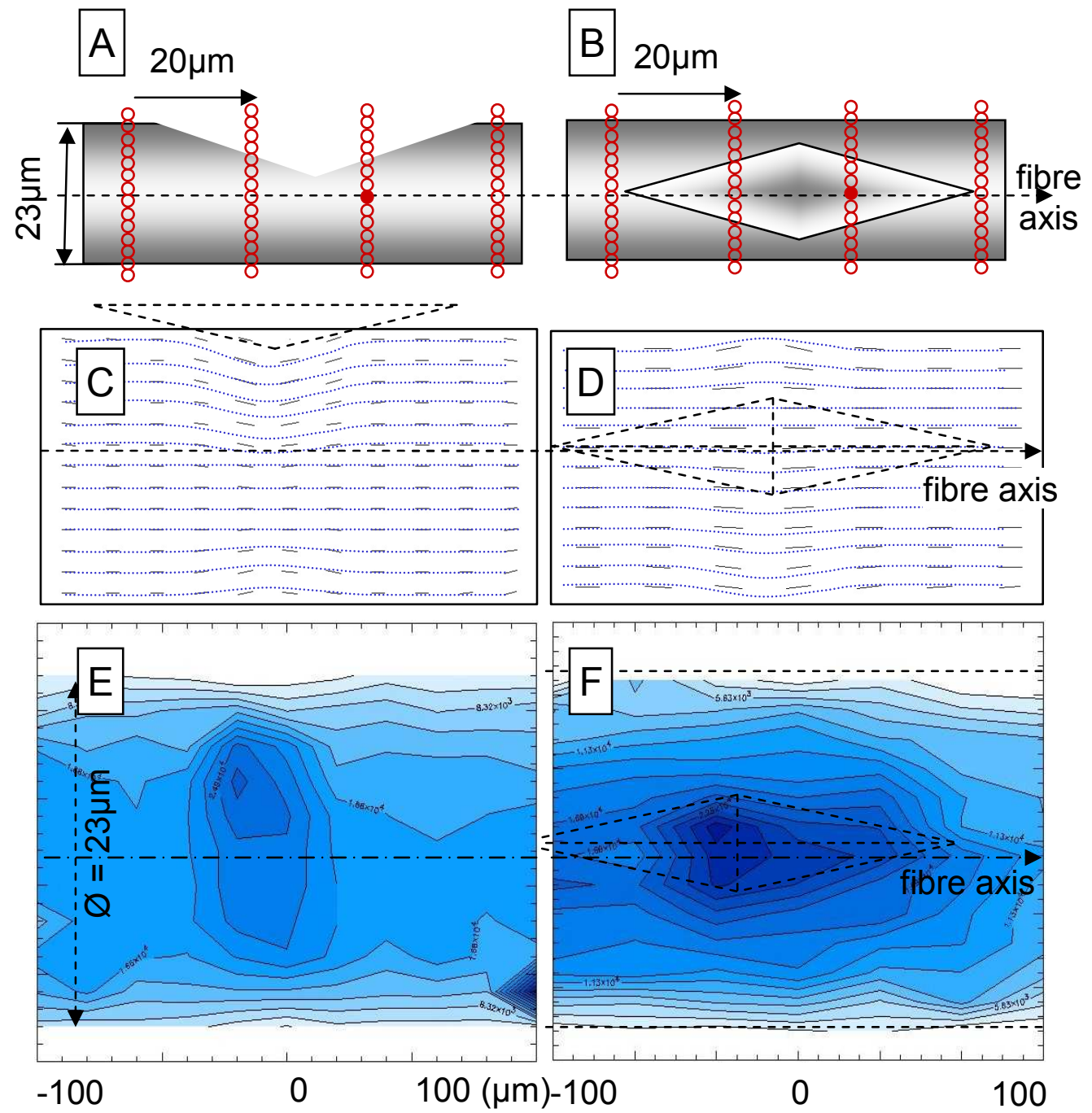

http://mc.manuscriptcentral.com/pm-pml 

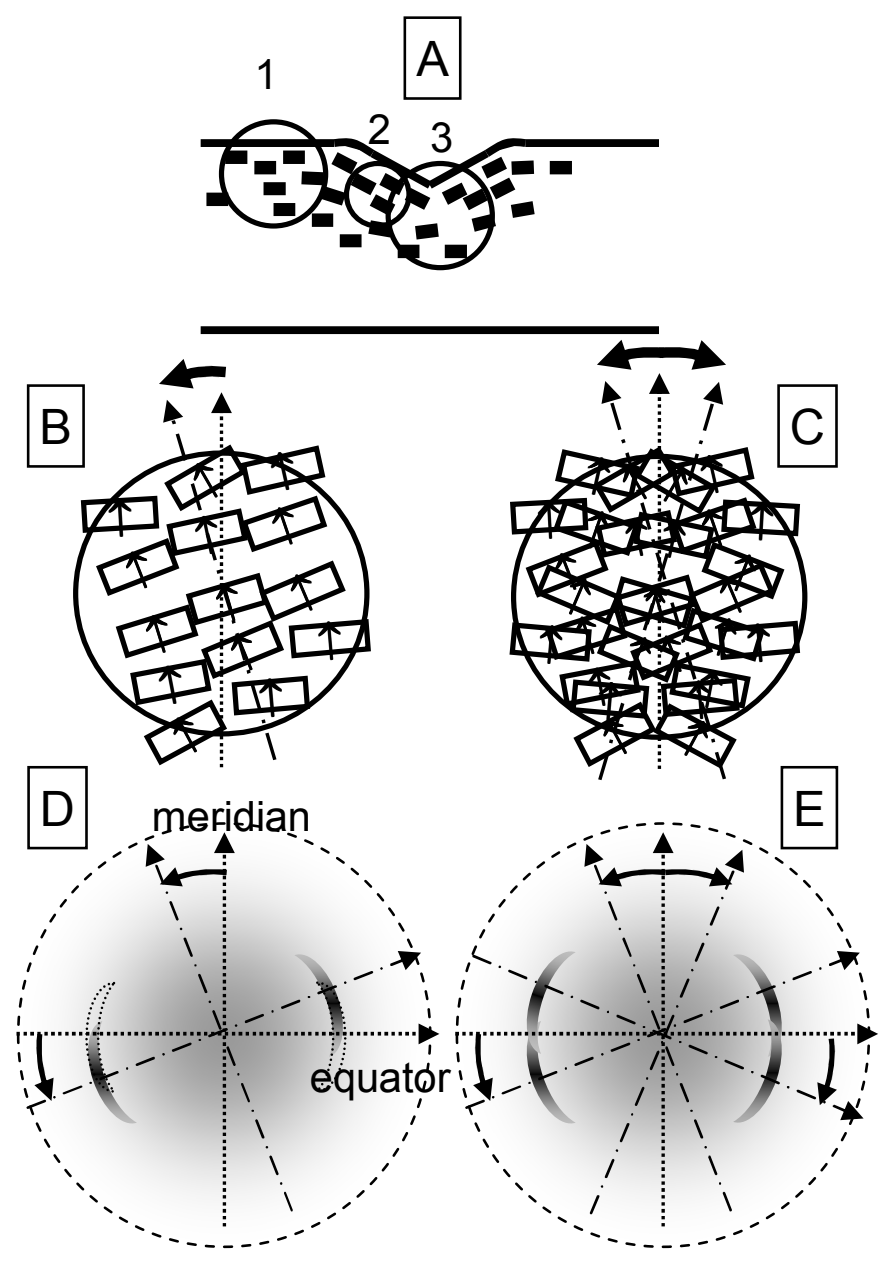